Physics in Collision (PIC 2013)

International Journal of Modern Physics: Conference Series

Vol. 31 (2014) 1460295 (5 pages)

(C) The Author

DOI: $10.1142 / \mathrm{S} 2010194514602956$

\title{
Performance and operation experience of the ATLAS Semiconductor Tracker
}

\author{
Zhijun Liang \\ (On Behalf of the ATLAS Collaboration) \\ University of Oxford \\ zhijun.liang@cern.ch
}

Published 15 May 2014

\begin{abstract}
We report on the operation and performance of the ATLAS Semi-Conductor Tracker (SCT), which has been functioning for 3 years in the high luminosity, high radiation environment of the Large Hadron Collider at CERN. We also report on the few improvements of the SCT foreseen for the high energy run of the LHC. We find $99.3 \%$ of the $\mathrm{SCT}$ modules are operational, the noise occupancy and hit efficiency exceed the design specifications; the alignment is very close to the ideal to allow on-line track reconstruction and invariant mass determination. We will report on the operation and performance of the detector including an overview of the issues encountered. We observe a significant increase in leakage currents from bulk damage due to non-ionizing radiation and make comparisons with the predictions.
\end{abstract}

\section{Introduction}

The ATLAS detector ${ }^{1}$ is a multi-purpose apparatus designed to study a wide range of physics processes at the Large Hadron Collider (LHC) at CERN. The recent observation of a Higgs-like particle reported by the ATLAS and CMS collaborations is a milestone in particle physics history. ${ }^{2,3}$ The study of the Higgs-like particle rely heavily on the excellent performance of the ATLAS inner detector tracking system. The semiconductor tracker (SCT) is a precision silicon microstrip detector which forms an integral part of this tracking system. The SCT is constructed of 4088 silicon detector modules, ${ }^{4,5}$ for a total of 6.3 million strips. Each module operates as a stand-alone unit, mechanically, electrically, optically and thermally. The modules are mounted into two types of structures: one barrel, made of 4 cylinders, and two end-cap systems made of 9 disks each. The SCT silicon micro-strip sensors ${ }^{6}$ are processed in the planar p-in-n technology. The signals are processed in the front-end ABCD3T ASICs, ${ }^{7}$ which use a binary readout architecture. Data is transferred to the off-detector readout electronics via optical fibres.

This is an Open Access article published by World Scientific Publishing Company. It is distributed under the terms of the Creative Commons Attribution 3.0 (CC-BY) License. Further distribution of this work is permitted, provided the original work is properly cited. 


\section{Detector Operations}

The SCT was installed into ATLAS, and was ready for the first LHC proton-proton collisions at a centre of mass energy of $7 \mathrm{GeV}$ in March 2010. Since then and until the end of running in February 2013, more than 99\% of the 6.3 million readout channels were operational. During operation several enhancements were introduced into the SCT Data Acquisition System (DAQ) ${ }^{8}$ in order to avoid potential sources of inefficiency. SCT introduced online monitoring of chip errors in the data and the automatic reconfiguration of the modules with errors. In addition, an automatic global reconfiguration of all SCT module chips every 30 minutes was implemented. There are 90 RODs in the SCT DAQ each of which processes the data for up to 48 modules, if a ROD experiences errors it will exert a BUSY signal which prevents all ATLAS sub-systems from recording data. From 2011 RODs exerting a busy were automatically removed, reconfigured, and reinserted into a run thus having a minimal impact on data taking.

\section{Hit Efficiency}

A high intrinsic hit efficiency is crucial for the operation of the SCT. The intrinsic efficiencies of the SCT modules are measured by extrapolating well-reconstructed tracks through the detector and counting the number of hits on the track and 'holes' where a hit would be expected but it is not found. Dead channels were removed from the analysis. The efficiency is higher than $99 \%$ and the fraction of disabled strips in each layer is less than $0.6 \%$ as shown in Fig. 1(a).

To maintain the high hit efficiency and reduce fake hits, dedicated readout timing calibrations were performed during the collisions runs. The in-time hit efficiency as a function of readout delay time has been studied for each SCT module as shown in Fig. 1(b). The readout timing of 4088 modules were optimized and synchronized to 2 ns precision level.

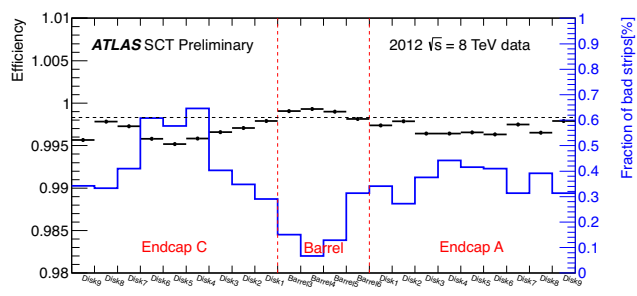

(a)

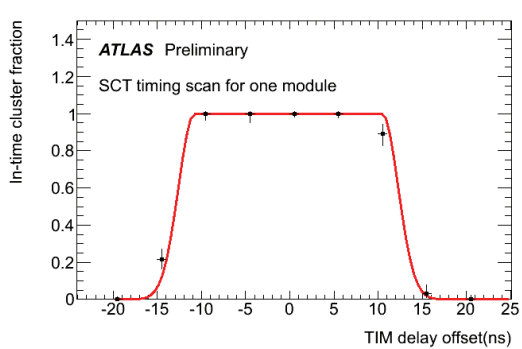

(b)

Fig. 1. (a) The SCT hit tracking efficiency (black). The blue line and right-hand axis indicates the fraction of disabled strips in each layer. (b) The in-time hit efficiency as a function of readout delay time for one SCT module recorded during a SCT timing calibrations in collisions run. 


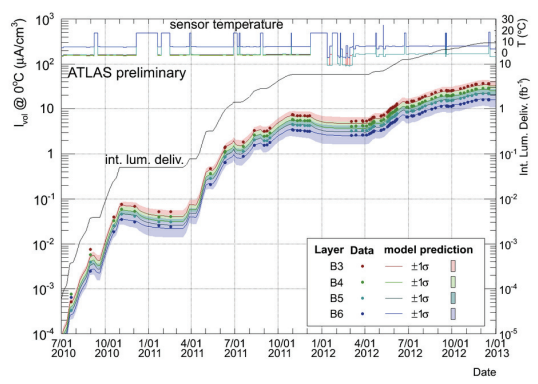

Fig. 2. SCT barrel leakage currents during 2010, 2011 and 2012, showing correlations with delivered luminosity and temperature, and compared to predictions from Monte Carlo

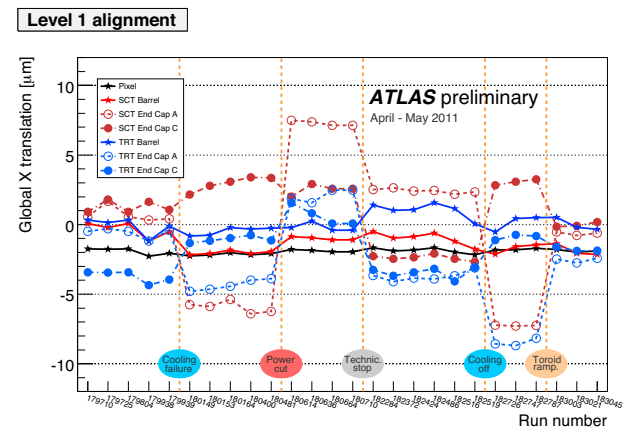

(a)

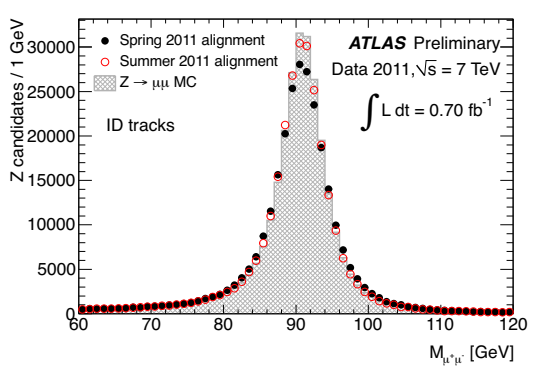

(b)

Fig. 3. (a) Subsystem level, Level One, alignment corrections performed on a run by run basis starting from a common set alignment constants. The corrections shown are for translations in the global $\mathrm{x}$ direction in different data taking runs. (b) Invariant mass distribution of $Z \rightarrow \mu \mu$ decays, where the mass is reconstructed using track parameters from the Inner Detector track of the combined muons only, using about $702 p b^{-1}$ of data collected during spring 2011. Ideal alignment performance based on Monte Carlo is compared to observed performance of data processed with spring 2011 alignment and data processed with updated alignment constants.

\section{Radiation Damage}

Radiation damage was a key consideration during the design phase of the SCT. Irradiation of silicon sensors results in damage in the bulk silicon and the dielectric layers, with main effects so far being the increase in leakage current of the sensor. Therefore the leakage current has been careful measured during the operation. Fig. 2 shows these measurements as a function of time, along with the integrated luminosity delivered by the LHC on the same time scale. The measured values of the fluence and leakage current are in agreement with predictions from a FLUKA based simulation. ${ }^{9}$ 

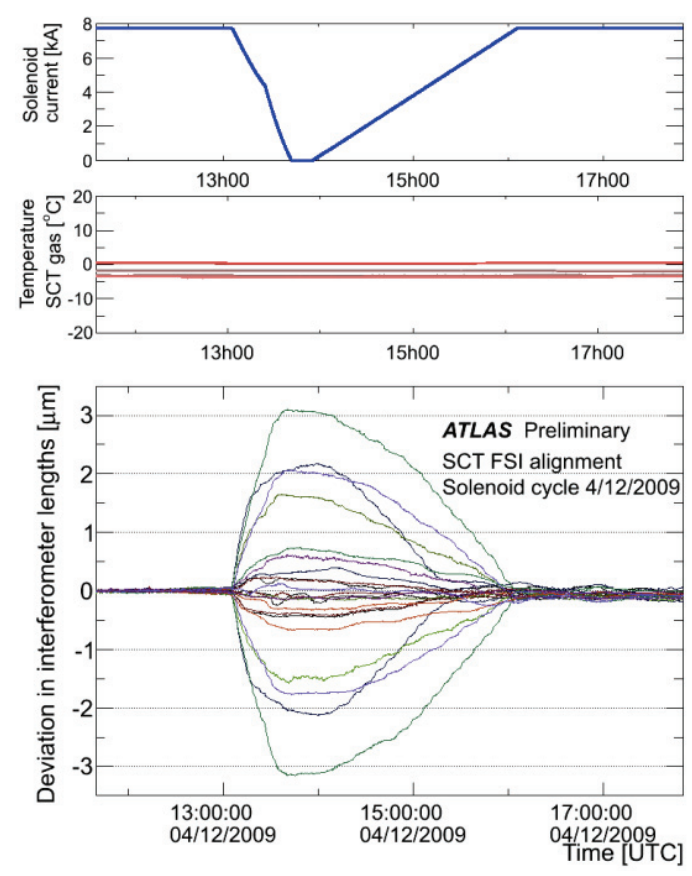

Fig. 4. The effect was observed in the FSI during ramping of the solenoid magnetic field in December 2009. The upper plots show the current in the magnets and the temperature of the gas in the SCT; the lower plot shows the detector movements monitored by different laser lines.

\section{Alignment}

\subsection{Track based alignment}

Alignment is performed using an algorithm which minimises the the $\chi^{2}$ between the the measured hit position and the expected position based on on track extrapolation. The alignment is performed at 3 different levels of granularity corresponding to the mechanical layout of the detector: Level 1 corresponds to entire sub-detector barrel and end-caps of Pixel Detector, SCT and TRT. Level 2 deals with silicon barrels and discs, TRT barrel modules and wheels. Level 3 aligns each silicon module and TRT straws having 700,000 degrees of freedom in total. Level 1 alignment corrections performed on a run by run basis starting from a common set alignment constants. As shown in Fig. 3(a) large movements of the detector are measured from track based alignment after hardware incidents. In between these periods, little $(<1 \mu \mathrm{m})$ movement is observed indicating that the detector is generally very stable.

After performing three levels of alignment, excellent agreement was found in the residual distributions for both barrel and end cap. The resolution in the $Z \rightarrow \mu \mu$ invariant mass distribution from reconstructed tracks, which is shown in Fig. 3(b), is close to the Monte-Carlo expectation. 


\subsection{Laser alignment}

The alignment stability is monitored using a Frequency Scanning Interferometry (FSI) system, which uses data from interferometers attached to the SCT structure to measure changes in length with submicron precision. Fig. 4 shows the effect observed in the FSI during ramping of the solenoid magnetic field in December 2009. Displacements up to $3 \mu \mathrm{m}$ are observed while the field is ramping but the detector is observed to return to its initial position at the end of the ramping process.

One of the most important result from FSI measurements is that the SCT detector is found to be stable at the $\mu \mathrm{m}$ level over extended periods of time, in agreement with the track-based alignment results.

\section{Summary}

After three years of operation, the SCT is performing well within its design specification in highly challenging conditions. More than $99 \%$ of the detector is still operational. Radiation damage has been observed and is well described by simulation. The detector alignment is very close to the ideal to allow on-line track reconstruction and invariant mass determination.

\section{References}

1. The ATLAS Collaboration, JINST 3, S08003 (2008).

2. The ATLAS Collaboration, Phys. Lett. B716, 1 (2012).

3. The CMS Collaboration, Phys. Lett. B716, 30 (2012).

4. A. Abdesselam et al., Nucl. Instrum. Meth. A568, 642 (2006).

5. A. Abdesselam et al., Nucl. Instrum. Meth. A575, 353 (2007).

6. J. Carter et al., Nucl. Instrum. Meth. A578, 98 (2007).

7. F. Campabadal et al., Nucl. Instrum. Meth. A552, 292 (2005).

8. A. Barr et al., JINST 3, P01003 (2008).

9. A. Ferrari, P. R. Sala, A. D. Fasso, J. Ranft, CERN-2005-010. 